\title{
Experimental analysis of the use of wet porous media for thermal protection against high intensity heat fluxes
}

\author{
A.R. Figueiredo, J.J. Costa * \\ Departamento de Engenharia Mecânica, Universidade de Coimbra-Polo II, 3030 Coimbra, Portugal
}

Received 20 March 2003; received in revised form 15 July 2003

\begin{abstract}
An experimental technique is implemented to analyse the behaviour of a wet porous medium under the direct impact of a flame, and the possibility of its use as a thermal protection device. The data obtained show that relatively thin and light thermal protections, with moderate values of water consumption, can be developed, presenting high levels of protection. The presentation of the data in a dimensionless form provides a useful tool to estimate the thickness and the water content of the protective device that are needed for expected values of incident heat flux and prescribed time of protection.
\end{abstract}

(C) 2003 Elsevier Ltd. All rights reserved.

\section{Introduction}

The study of porous media is a very important field of research in several areas of engineering, as agricultural, chemical, mechanical, as well as in geophysics and physics of soils. Porous media have been traditionally applied as thermal insulation materials. It is also known that moist fire-protection materials show good characteristics, the latent heat of water playing an important role in the resistance against heat propagation. These materials are usually made of mixtures of cement mortar and high-water content materials such as silica gel or moist perlites [1]. A different concept for an active thermal insulation system was proposed by Maruyama et al. [2], using a semitransparent porous medium and injecting a low-temperature gas. The porous medium heated by an intense irradiation is effectively cooled by the gas injected from the back face of the layer, and the heated gas is used for transpiration cooling of the front surface. The same authors have presented a heat transfer model and investigated the transient characteristics of the active thermal insulation layer [3] and the effect of the injection gas [4]. The possibility of controlling the

\footnotetext{
${ }^{*}$ Corresponding author. Tel.: +351-239-790732; fax: +351239-790771.

E-mail address: jose.costa@dem.uc.pt (J.J. Costa).
}

radiation heat transfer by the active thermal insulation layer and its similarity to that of a triode have also been studied [5]. In a subsequent work, Maruyama and Shimizu [6] reported the measurements of the transient and the steady-state temperature distribution in an active thermal insulation layer heated by a gas burner, and the results were compared with a numerical solution. One field where thermal protection has a vital interest is forest-fire fighting [7-10]. Over the past 25 years, recurring burnovers around the world have resulted in an ongoing effort on numerous fronts to improve the safety of fire fighters and trucks. During a fire-particularly a forest fire-levels of thermal radiation or convection as high as 160 and $110 \mathrm{~kW} / \mathrm{m}^{2}$, respectively, can be reached and prevail during an interval of time of 2-5 min. Experience has demonstrated that the traditional use of individual fire shelters, made of a highly reflexive aluminium foil material, or of water sprinklers, often is not an absolute guaranty of survival [11]. In particular, the material of fire shelters resists very well and shows good protection characteristics under high fluxes of thermal radiation, provided that fire fighters wear gloves to handle them. However, it degrades quickly when flames impinge it directly, and is no longer suitable for fire protection under burnover conditions. This is the fundamental reason why the present study is especially addressed to the convective heat transfer resulting from the impact of a flame on a thermal shield. 


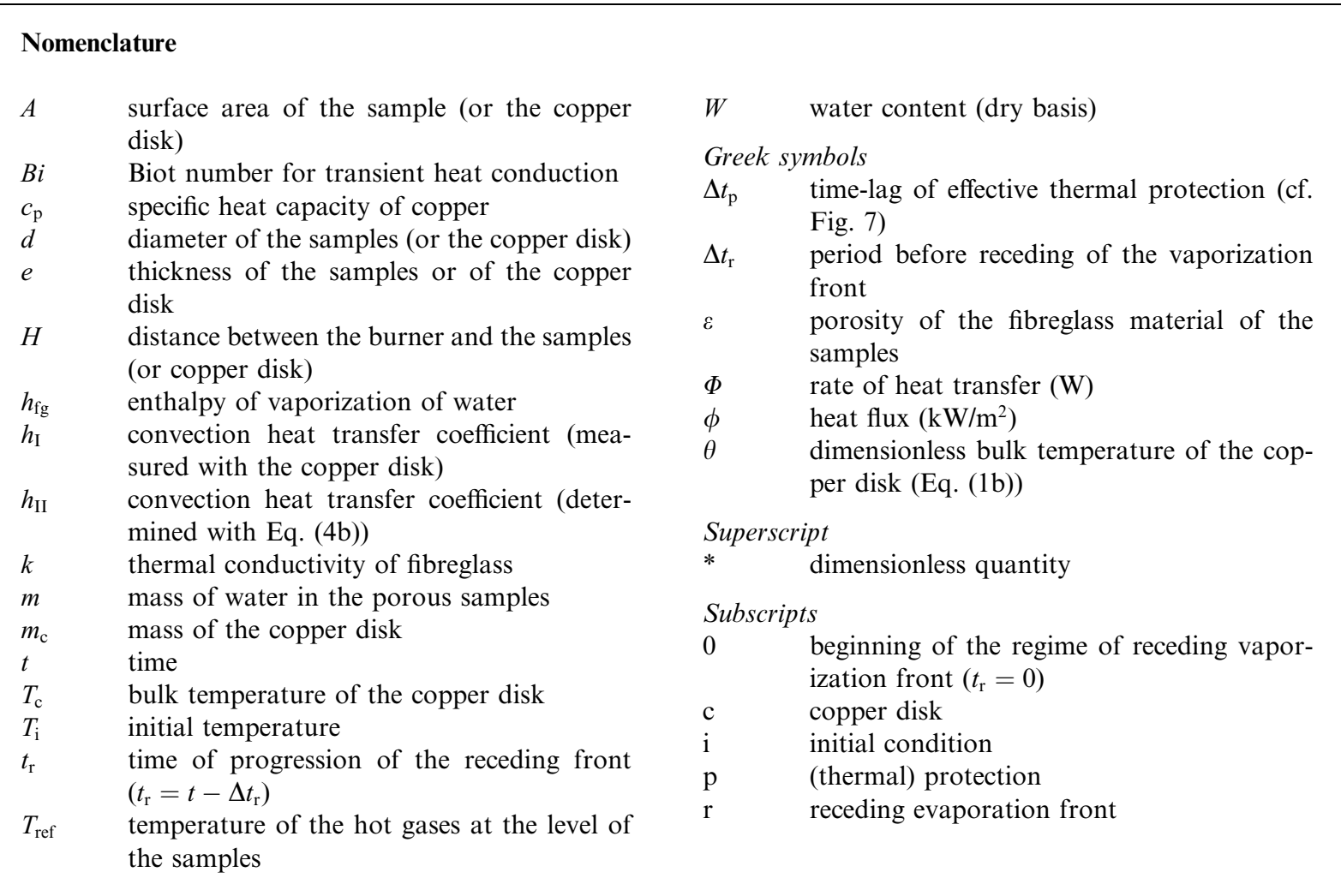

Although some well established theories for the simultaneous heat and mass transfer in saturated or unsaturated porous media have been successfully applied to describe the reaction of a porous medium to an external thermal solicitation [12-14], the equations obtained by application of the conservation principles for mass, energy and momentum are very non-linear and, in most cases, a numerical technique is necessary to solve them. Many times there is also a lack of complete knowledge about the values of the coefficients present in the equations, which must be taken nearly always as experimental facts [15]. In such conditions, the use of simplified models together with an experimental approach is the only available way to treat the problem. It is well known that, for drying operations, a mass diffusion model based on Fick's law often gives results with great practical interest [16]. On the other hand, for drying processes at high temperatures, the so-called receding evaporation front model describes the process with enough precision, even if in both precedent cases the underlying physics is not always completely understood [17].

In this paper, an experimental study concerning the analysis of the behaviour of a wet porous/fibrous medium under the direct action of a flame is presented. In order to minimise the influence of thermal radiation, which was neglected in the experiments, use was made of an aluminium foil covering the frontal surface of the samples, and a small thickness, low-emissivity flame was used. The thermal protection effect is based on the consumption of the absorbed heat to evaporate water that is contained in a multi-layer sample of a wet porous medium, placed horizontally over a non-luminous flame.

The ultimate goal of the work is to analyse the possibility of the use of such a system as thermal protection to improve the levels of fire line safety.

\section{Experimental apparatus and procedure}

The experimental set-up is schematically represented in Fig. 1. The main components were an electronic balance, with a precision of $0.01 \mathrm{~g}$, a set of k-type thermocouples $(0.2 \mathrm{~mm}$ diameter) and a computer with suitable programs for data acquisition and treatment of the temperature and mass time evolutions. The samples to be analysed were made of superimposed fibreglass layers, with the shape of $65 \mathrm{~mm}$ diameter disks, as illustrated in Fig. 2(a) and (b). Each fibreglass circular layer was cut from a fibreglass "mate-type" foil, with a specific weight of about $460 \mathrm{~g} / \mathrm{m}^{2}$, as indicated by the manufacturer. The external surface of each sample, directly exposed to the flame, was a disk with the same diameter made of aluminium foil (approximately 0.1 $\mathrm{mm}$ thick), a material that is currently used in fire 


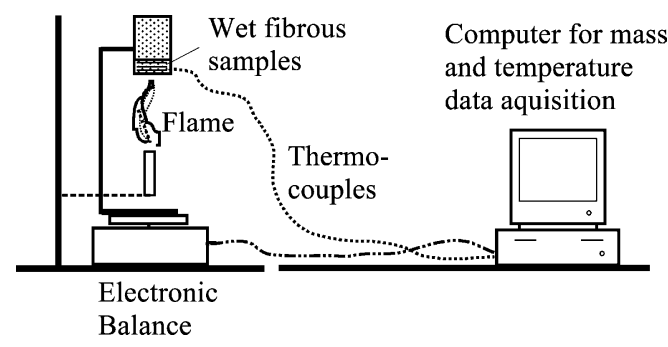

Fig. 1. Overall sketch of the experimental installation.

shelters and presents a low emissivity $(10 \%$ is a value often indicated in the bibliography on fire shelters). After mounting, the diameter of the external exposed surface is $63 \mathrm{~mm}$. To allow the water vapour exhaust from the porous sample, the external aluminium foil was previously perforated in all experiments with evenly distributed holes of $0.1 \mathrm{~mm}$ diameter, corresponding to a surface density of about 17,000 holes $/ \mathrm{m}^{2}$. Three pairs of thermocouples were placed within the samples, as shown in Fig. 3(a). Pair no. 1 was placed immediately after the aluminium foil, and its temperature was taken as representative of the temperature of the external surface of the sample. Pair no. 2 measured the temperature at an intermediate depth inside the sample, and pair no. 3 indicated the temperature of the surface of the Bakelite plate, in the back of the sample. This Bakelite plate, 1.5 $\mathrm{mm}$ thick, represented the surface of a body to be protected from the impact of the flame and was supposed to be adiabatic and impermeable. A schematic representation of these details is shown in Fig. 3, together with the main geometrical variables.

Before the experiments, a certain number of properties of the fibreglass used to make the samples was measured, namely: the thickness, the dry weight, the saturation water content and the global porosity. The electronic balance was used to measure the dry weight of each sample, as well as its water content. Maximum saturation was achieved for water contents in the range $70 \%-110 \%\left(W_{\mathrm{i}}\right.$, dry basis). The porosity of the material was determined under saturation conditions, by dividing the volume of the absorbed mass of water by the total volume of the samples. The values obtained for five different disks are summarized in Table 1, together with the corresponding average values.
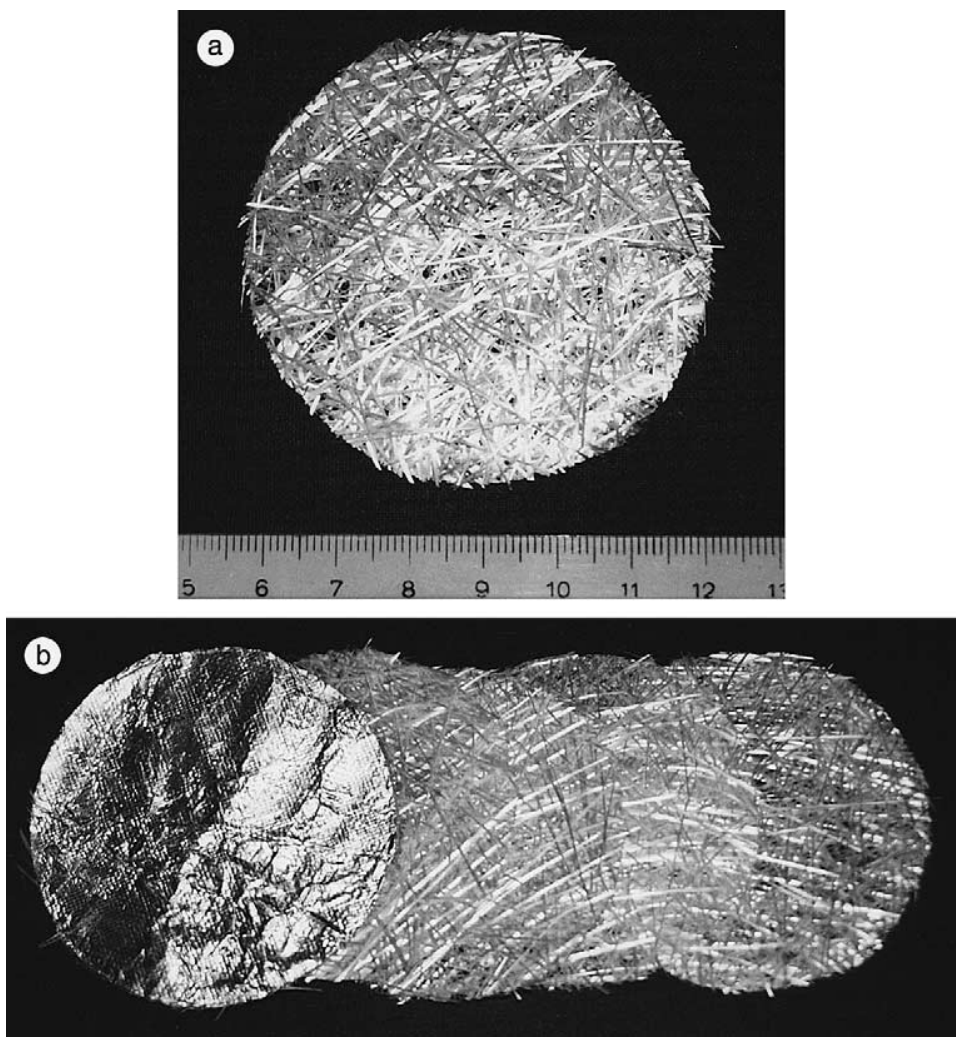

Fig. 2. Illustrations of a typical sample used in the experiments: (a) a single layer made of "mate-type" fibreglass foil; (b) expanded view of the superimposed fibreglass layers, covered with a disk of perforated aluminium foil. 


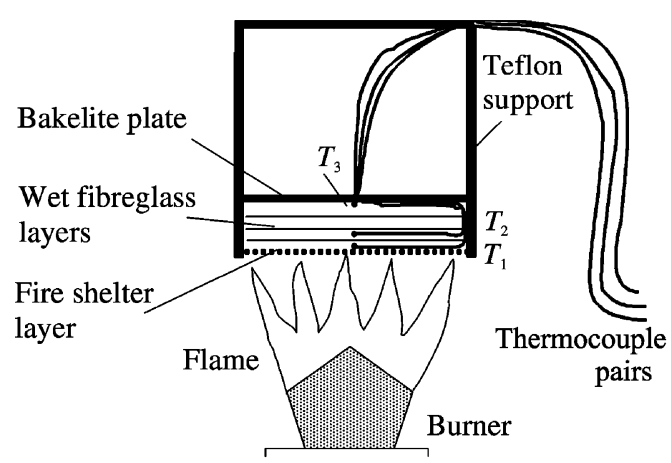

(a)

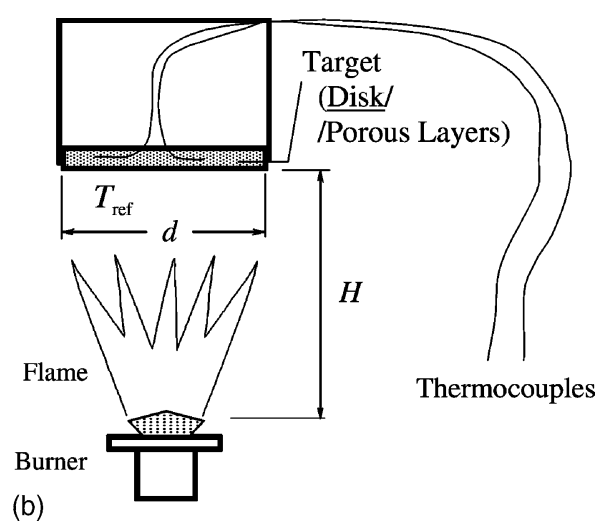

Fig. 3. Schematic drawing of (a) the porous sample with the emplacement of three thermocouple pairs, and (b) the main geometrical characteristics. (The exposed surface of the target/sample has a diameter $d=63 \mathrm{~mm}$.)

Table 1

Dimensions, dry weight, water content and porosity of the fibreglass disks

\begin{tabular}{llllcl}
\hline $\begin{array}{l}\text { Sample } \\
\text { no. }\end{array}$ & $\begin{array}{l}e / \mathrm{mm} \\
\text { (approx.) }\end{array}$ & $\begin{array}{l}D / \\
\mathrm{mm}\end{array}$ & $\begin{array}{l}\text { Dry } \\
\text { weight/g }\end{array}$ & $\begin{array}{l}W_{\mathrm{i}} / \% \\
\text { (dry basis) }\end{array}$ & $\varepsilon / \%$ \\
\hline 1 & 0.7 & 65 & 1.53 & 84 & 56 \\
2 & 0.7 & 65 & 1.59 & 107 & 74 \\
3 & 0.65 & 65 & 1.58 & 84 & 62 \\
4 & 0.6 & 65 & 1.34 & 106 & 71 \\
5 & 0.7 & 65 & 1.57 & 110 & 75 \\
Average & 0.67 & 65 & 1.52 & 98.2 & 67.6 \\
\hline
\end{tabular}

The flame was produced by the combustion of an air-butane pre-mixture in a camping stove gas burner, which could be set at different fuel rates. The flame was nonluminous, with small thickness and emissivity, impinging on a highly reflective surface (aluminium foil). Thus the flame was assumed to be nonparticipating and radiation was neglected in all the experiments, as also reported by other authors in a previous work on the subject [6].

The samples were directly placed above the vertical flame, at different distances $(H)$ from the burner, as it is sketched in Fig. 3. During each experiment, the time evolutions of the mass and of the internal temperatures of the sample, at different depths, were measured and stored for later treatment.

For each run, the temperature of the flame was measured at the horizontal location of the outer surface of the samples and on the vertical axis of symmetry of the system. After correction for the radiation effects, this value was assumed as the reference temperature $\left(T_{\text {ref }}\right)$ of the hot gas impinging flow.

A great number of tests was performed in the following ranges of conditions: burner-to-sample distance, $H=35-140 \mathrm{~mm}$; water content, $W_{\mathrm{i}}=0 \%-110 \%$. In the present paper, however, the experiments corresponding to initial saturation conditions ( $\left.W_{\mathrm{i}}=70 \%-110 \%\right)$ will be particularly addressed, the main characteristics of which being summarized in Table 2 .

No correlations were found in the literature to characterise the thermal interaction between a flame and a horizontal surface, in any situation completely similar to the one studied here [18]. A standard method (capacitance calorimeter method), based on the increase of the bulk temperature of a thin copper disk $(e=5 \mathrm{~mm}$; $m_{\mathrm{c}}=139.7 \mathrm{~g}$ ), with the same external area and placed at the same location as the samples in the experiments (see Fig. 3(b)), was then used for the calculation of the heat transfer coefficients $[19,20]$. In these conditions ( $B i<0.1$, i.e., negligible resistance to the internal heat conduction), the time evolution of the copper disk temperature may be described, in dimensionless form, by

$$
\ln (\theta)=-\frac{h A}{m_{\mathrm{c}} c_{\mathrm{p}}} \cdot t
$$

Table 2

Main characteristics of the tests performed in the apparatus with saturated samples

\begin{tabular}{lllrr}
\hline $\begin{array}{l}\text { Run } \\
\text { no. }\end{array}$ & $\begin{array}{l}\text { Sample } \\
\text { thickness, } \\
e / \mathrm{mm}\end{array}$ & $\begin{array}{l}\text { Number of } \\
\text { fibreglass } \\
\text { layers }\end{array}$ & $W_{\mathrm{i}} / \%$ & $H / \mathrm{mm}$ \\
\hline 1 & 4 & 6 & 92 & 70 \\
2 & 2 & 3 & 117 & 70 \\
3 & 2.7 & 4 & 96 & 70 \\
4 & 4 & 6 & 70 & 70 \\
5 & 2.7 & 4 & 85.5 & 140 \\
6 & 2.7 & 4 & 89 & 110 \\
7 & 2.7 & 4 & 87 & 110 \\
8 & 2.7 & 4 & 96 & 110 \\
9 & 2.7 & 4 & 90 & 35 \\
\hline
\end{tabular}


with

$\theta=\frac{T_{\mathrm{c}}(t)-T_{\text {ref }}}{T_{\mathrm{i}}-T_{\text {ref }}}$

The convection heat transfer coefficient can thus be estimated by previously determining the slope of the straight line plots of $\ln (\theta)$, and then

$h_{\mathrm{I}}=-\frac{m_{\mathrm{c}} c_{\mathrm{p}}}{A} \cdot \frac{\mathrm{d}}{\mathrm{d} t}(\ln \theta)$.

\section{Analysis of the results}

The mass evolutions represented in Fig. 4 were obtained for three different tests, performed with similar values of initial water content, thickness of the samples, fuel flow rate and distance $H$. It is seen that the curves display the same general trend and a good reproducibility. The same conclusions hold for the time evolution of dimensionless temperature of the copper disk, as it is apparent in Fig. 5: the experimental data, corresponding to two different runs with the copper disk, placed at a particular distance from the burner, are well correlated by straight lines with very similar slopes.

Fig. 6 represents the time evolutions of the internal temperature field of a dry sample and an unsaturated sample with initial water content of about $32 \%$. Both samples had the same thickness and were submitted to similar experimental conditions. The influence of the presence of water within the porous medium is apparent: although there is a steeper increase of temperature at the beginning of the experiment, due to the higher thermal conductivity of the wet sample (solid symbols in Fig. 6), a global reduction of the temperature levels can be observed. In Fig. 7, the time evolutions of both the mass of

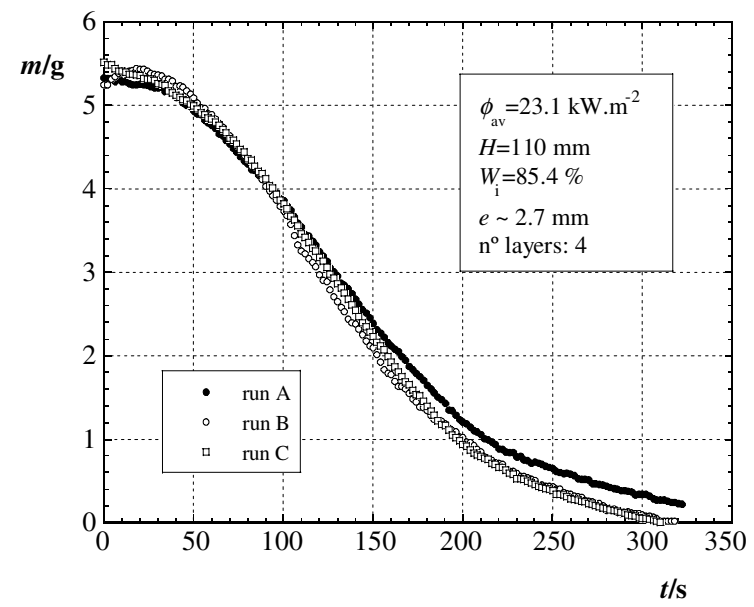

Fig. 4. Transient evolutions of the mass of water, obtained in three different experiments under similar conditions.

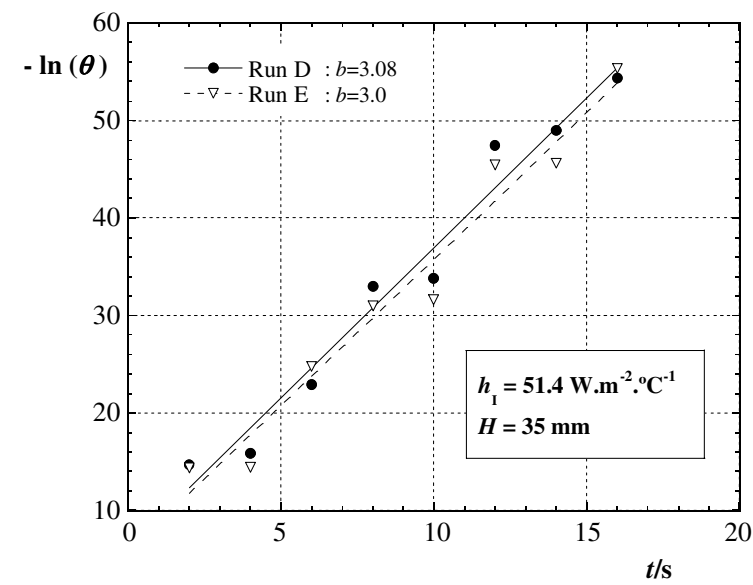

Fig. 5. Comparison of the increase of the copper disk dimensionless temperature, for two different tests under similar conditions ( $b$ : slope of the fitted lines).

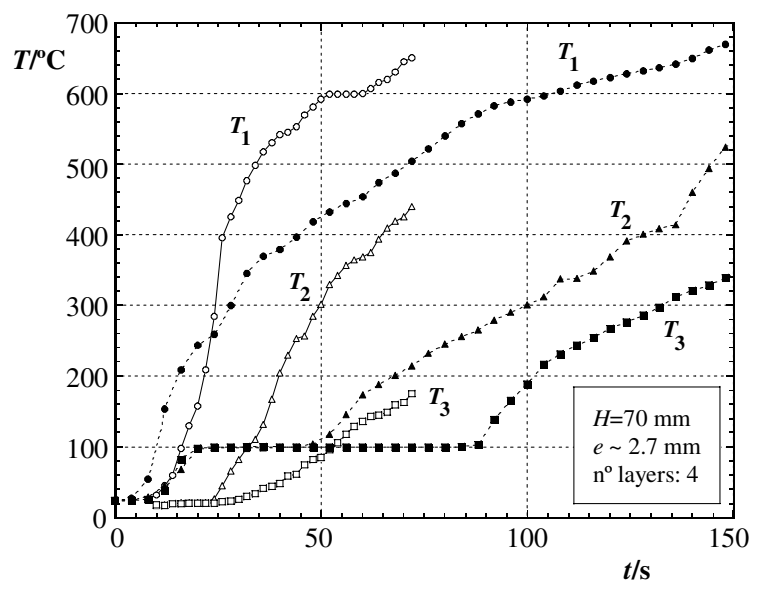

Fig. 6. Influence of the initial water content on the transient behaviour of a fibreglass sample, for two different tests under similar boundary conditions: open symbols - dry sample, $W_{\mathrm{i}}=0$; solid symbols-wet, unsaturated sample, $W_{\mathrm{i}}=32 \%$ (burner at full-rate).

water and of the temperature field are represented, for an initially saturated sample. Mass loss rate or drying kinetics curves can be obtained for all the experiments by deriving the mass evolutions in order to time, as illustrated in Fig. 8 for the same experiment (no. 3).

In all experiments with initially saturated samples, the temperature field showed a pattern similar to the one of Fig. 7. Its observation strongly suggests that, after a short warming-up period at the end of which the temperature of the sample globally attains $100{ }^{\circ} \mathrm{C}$, a receding front of vaporization is developed within the porous sample. This receding front regime begins when the temperatures indicated by the thermocouple pair 


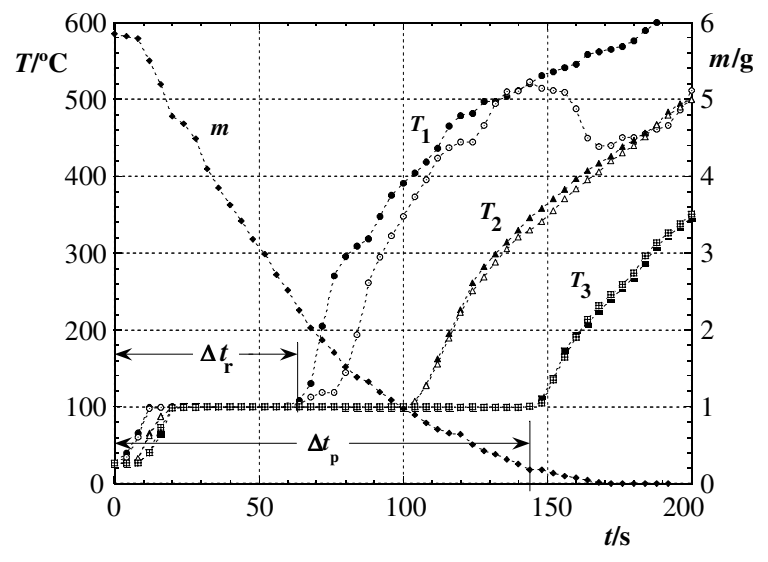

Fig. 7. Time evolutions of the internal temperature field and of the global water mass of the sample, as measured in run no. 3 (see Tables 2 and 3): $H=70 \mathrm{~mm}, e=2.7 \mathrm{~mm}, 4$ layers, $W_{\mathrm{i}}=96 \%, \phi=43 \mathrm{~kW} / \mathrm{m}^{2}$.

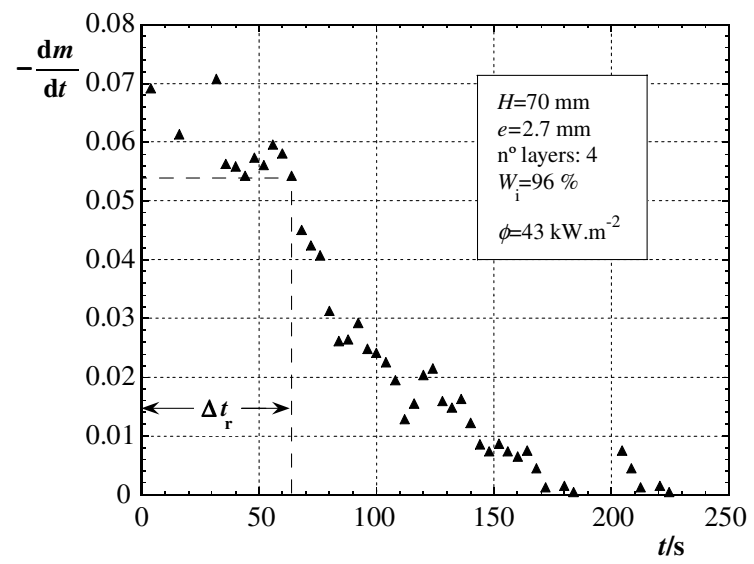

Fig. 8. Time evolution of the mass rate of water contained in the porous sample, during experiment no. 3 (see Tables 2 and 3 ).

no. 1 start increasing above the $100{ }^{\circ} \mathrm{C}$ plateau. In fact, while the porous medium is saturated, water may be charged to the heated surface by the action of capillarity and gravity, as well as by the effect of vapour formation. It is supposed that the capillary effects in the vertical direction are negligible, in view of the predominant fibre orientation (horizontal). Likewise, gravity is supposed to be unimportant due to the reduced vertical dimension of the samples $(2-3 \mathrm{~mm})$. Thus the vapour formation is probably the most significant mechanism of charging the liquid phase to the heated surface, during the early stages of the experiments $\left(t<\Delta t_{\mathrm{r}}\right)$. In these conditions, the feeding of liquid water and the formation of small droplets in the tiny holes of the surface are responsible for an increase of the external heat transfer area, with an associated increase of the absorbed heat rate. Only after having lost about $2 / 3$ of the initial water, a true vaporization front begins to recede into the sample. At that instant, $t=\Delta t_{\mathrm{r}}$, the plane surface area $A$ can be taken as a representative value for the heat transfer area and, as the temperature of the sample still is $100{ }^{\circ} \mathrm{C}$, all the absorbed heat is consumed in the vaporization of water. The thermal power transferred to the sample can then be estimated by multiplying the absolute value of the rate of water loss by the enthalpy of vaporization $h_{\mathrm{fg}}$ :

$\Phi=\left|\frac{\mathrm{d} m}{\mathrm{~d} t}\right|_{0} \times h_{\mathrm{fg}}$.

Accordingly, the heat flux and the convection heat transfer coefficient between the flame and the external surface of the sample can then be evaluated by using the following expressions, respectively:

$\phi=\frac{\Phi}{A}$,

$h_{\mathrm{II}}=\frac{\phi}{\left(T_{\mathrm{ref}}-100\right)}$.

The transient temperature field in Fig. 7 proves that the Bakelite plate is effectively protected from the incident flame, as long as its temperature does not exceed $100{ }^{\circ} \mathrm{C}$. This time interval will be hereafter designated as time-lag or time of protection and denoted by $\Delta t_{\mathrm{p}}$.

The results obtained in all considered experiments are summarized in Table 3, namely: the time-lag, $\Delta t_{\mathrm{p}}$, the surface heat rate, $\Phi$, and flux density, $\phi$, as well as the convection heat transfer coefficient determined by both above-described methods, $h_{\mathrm{I}}$ and $h_{\mathrm{II}}$. The maximum relative difference between $h_{\mathrm{I}}$ and $h_{\mathrm{II}}$ was of about $7 \%$. Incidentally, the values obtained for the heat transfer coefficient correspond to Biot numbers smaller than 0.1, which confirms that the hypothesis made for the thermal behaviour of the copper disk was a reasonable one.

It is possible to demonstrate that, when a vaporization front recedes within a porous medium, one can expect a linear relationship of the form $[15,17]$ :

Table 3

Summary of the relevant results obtained in the experiments with saturated samples

\begin{tabular}{llllll}
\hline Run no. & $\begin{array}{l}\text { Time- } \\
\text { lag, } \Delta t_{\mathrm{p}} / \mathrm{s}\end{array}$ & $\Phi / \mathrm{W}$ & $\begin{array}{l}\phi / \mathrm{kW} / \\
\mathrm{m}^{2}\end{array}$ & $\begin{array}{l}h_{\mathrm{I}} / \mathrm{W} / \\
\mathrm{m}^{2}{ }^{\circ} \mathrm{C}\end{array}$ & $\begin{array}{l}h_{\mathrm{II}} / \mathrm{W} / \\
\mathrm{m}^{2}{ }^{\circ} \mathrm{C}\end{array}$ \\
\hline 1 & 228 & 134 & 43 & 41.9 & 44.9 \\
2 & 130 & 124 & 39.7 & 41.9 & 41.6 \\
3 & 150 & 134.7 & 43 & 41.9 & 42.2 \\
4 & 192 & 129 & 41.4 & 41.9 & 43.3 \\
5 & 260 & 81.8 & 26.3 & 39 & 41 \\
6 & 300 & 69.4 & 22.3 & 36 & 42.4 \\
7 & 270 & 74.4 & 23.9 & 50 & 46.8 \\
8 & 210 & 96.7 & 31 & 49.7 & 51.7 \\
9 & 128 & 148 & 47.7 & 51.4 & 53.6 \\
\hline
\end{tabular}


$\alpha=\left[\left(\frac{\mathrm{d} m}{\mathrm{~d} t}\right)^{-2}+\frac{k}{h}\left(\frac{\mathrm{d} m}{\mathrm{~d} t}\right)^{-1}\right] \propto t$.

The parameter $\alpha$ is represented in Fig. 9 as a function of time for four different runs, corresponding all to similar values of the heat flux. It can be observed that a regime of a linear $\alpha \sim t$ relationship seems to exist in all the experiments. Furthermore, its beginning coincides with the instant $t=\Delta t_{\mathrm{r}}$ when the temperatures of the thermocouple pair no. 1 increase above $100{ }^{\circ} \mathrm{C}$ (compare the evolution of $\alpha$ for run no. 3 in Fig. 9 with the curve $T_{1}$ in Fig. 7). This relationship is represented in Fig. 10 for the

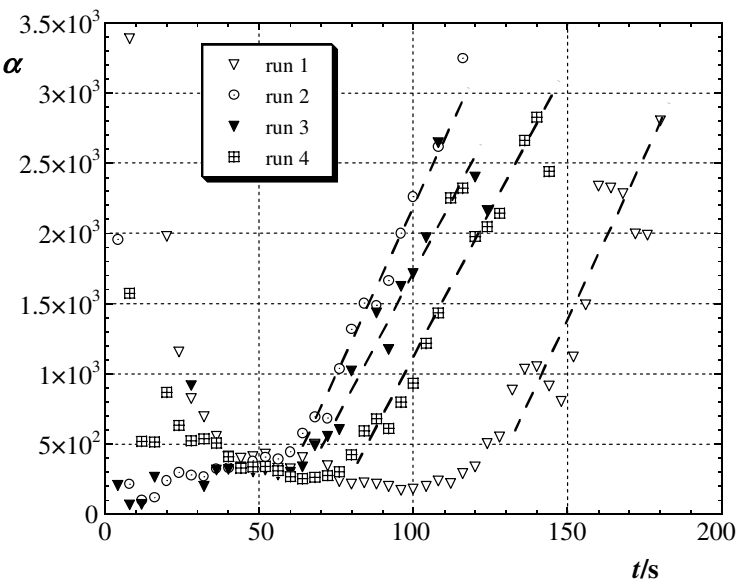

Fig. 9. Time variation of the parameter $\alpha$ (see Tables 2 and 3 for the test conditions).

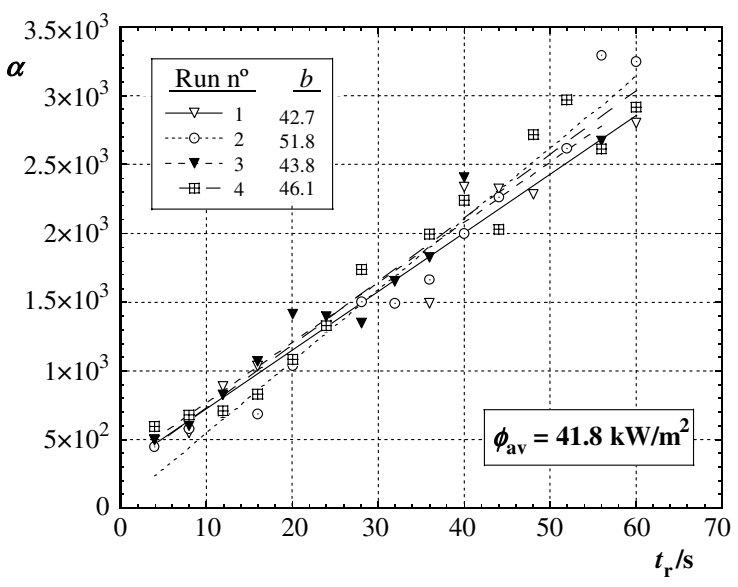

Fig. 10. Linear fittings to the time plots of the parameter $\alpha$, corresponding to the period of progression of the receding evaporation front, in four different tests (see Tables 2 and 3 ) and comparison of the respective slopes $b\left(t_{\mathrm{r}}=t-\Delta t_{\mathrm{r}}\right.$ in each experiment). same four runs, within the respective period of the "receding front regime", all data being well correlated by straight lines of similar slopes.

The fact that all experiments correspond to a characteristic behaviour of the porous medium allows the definition of a dimensionless drying curve, by choosing appropriate parameters, namely the dimensionless mass of water, $m^{*}$, and time, $t^{*}$, which are defined as, respectively:

$m^{*}=m / m_{\mathrm{i}}$,

$t^{*}=\frac{\Phi}{m_{\mathrm{i}} \cdot h_{\mathrm{fg}}} \cdot t$.

The time evolutions of the mass of water are plotted in Fig. 11(a), as measured in five experiments with different values of initial mass of water, absorbed heat flux, sample thickness and distance to the burner (see Tables
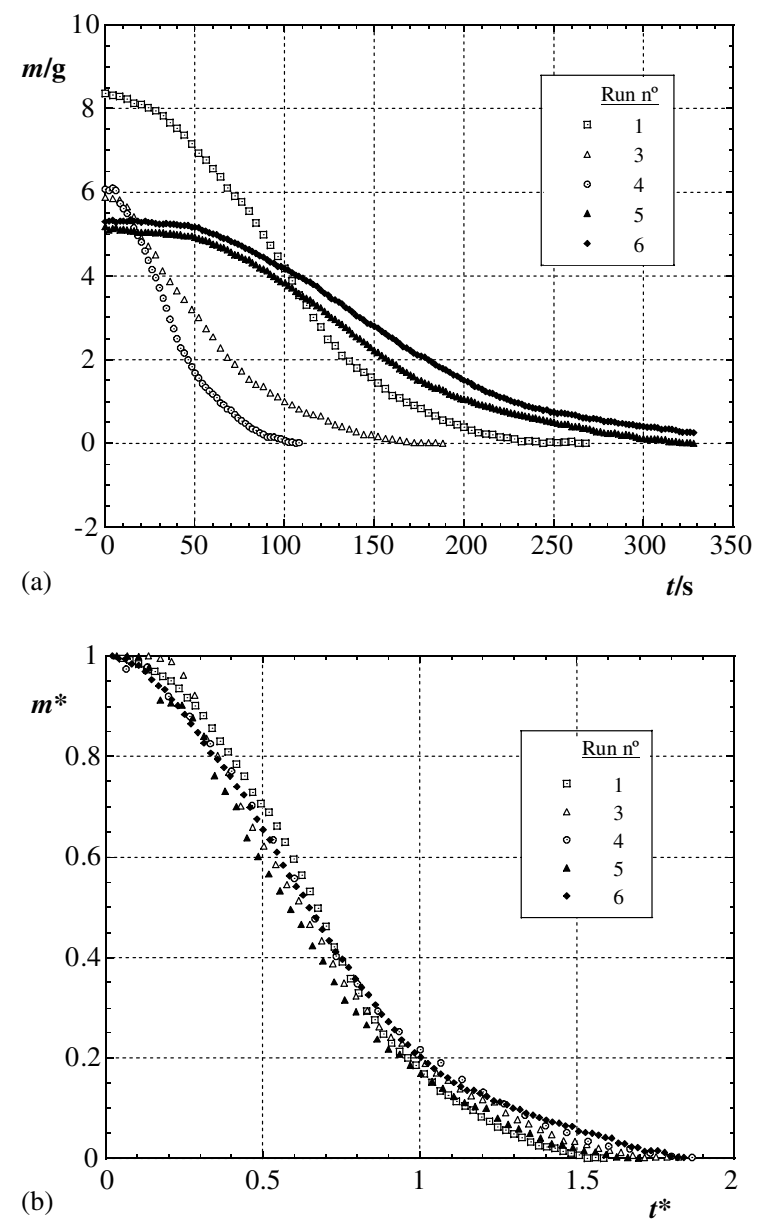

Fig. 11. (a) Dimensional and (b) dimensionless representations of the time variation of the mass of water, as measured in five experiments with different values of initial mass of water, $m_{\mathrm{i}}$, surface heat flux, $\phi$, and sample thickness, $e$ (see Tables 2 and 3). 
2 and 3). The corresponding dimensionless representations of Fig. 11(b) lead to self-similar profiles, describing a unique dimensionless law. For design purposes of any thermal protective device, made of some similar fibrous/ porous material, the relevance of the present study may be illustrated by the interpretation of that unique curve, as follows: (i) if the initial water content and the incident heat flux are known, it is possible to estimate through Eq. (7) an expected time-lag of thermal protection, $\Delta t_{\mathrm{p}}$; otherwise, (ii) for a prescribed time-lag, $\Delta t_{\mathrm{p}}$, and a certain level of heat transfer rate, $\Phi$, it is possible to calculate the required initial mass of water, $m_{\mathrm{i}}$, and thus ensuring the use of an appropriate thickness of the protective fibrous material.

\section{Conclusions}

In this work an experimental approach was implemented to assess the behaviour of a porous/fibrous medium, previously saturated with water, when submitted to the direct contact of a flame. It was shown that, in the interval of water content close to saturation conditions, the medium deployed a typical temperature field showing the existence of a particular regime, which is identified in the literature as the receding front of vaporization and has a relatively simple mathematical formulation.

It was also shown that a convenient treatment of the measured data enabled the direct determination of the absorbed heat flux for each experiment, and, subsequently, the value of the heat transfer coefficient between the surface and the flame. This result represents an important simplification of the experimental procedure, and works in fact as an original technique for the experimental determination of heat fluxes between a surface and an impinging flame. It was also possible to correlate the data with the so called time-lag or time of protection, defined as the interval of time during which the temperature of a protected surface does not exceed $100{ }^{\circ} \mathrm{C}$, in the interval of external heat fluxes analysed. It was also shown that the mass-time evolution of the analysed samples could be correlated by a unique characteristic curve of the drying kinetics by choosing suitable dimensionless parameters. This curve enables the determination of the time-lag of protection, if the values of the initial water content and of the incident heat flux are known. The described methodology can be of primary interest for future research and development of new porous materials to be used for thermal protection purposes. Results like those presented in this work can provide a valuable and straightforward design tool for more evolved configurations of protective devices against high intensity heat fluxes, if based on the same principle of absorption of phase change latent heat.
As a laboratory work to develop an experimental methodology for the assessment of the thermal protection capability of porous materials, it was not crucial for the moment to make an extensive characterisation of the hot gas flow. In a close future, this work will be extended to the use of impinging jet flames with controlled velocity, in order to get higher levels of incident heat fluxes and to compare results with published data and correlations.

\section{Acknowledgements}

This work was performed in the frame of the European Project SPREAD (project no. EVGI-CT-200100043). The authors are very grateful to the support of the project coordinator Prof. D. Xavier Viegas.

\section{References}

[1] Z.-F. Jin, Y. Asako, Y. Yamaguchi, M. Harada, Fire resistance test for fire protection materials with high water content, Int. J. Heat Mass Transfer 43 (24) (2000) 4395-4404.

[2] S. Maruyama, R. Viskanta, T. Aihara, Active thermal protection system against intense radiation, AIAA J. Thermo. Heat Transfer 3 (4) (1989) 389-394.

[3] S. Maruyama, T. Aihara, Transient behaviour of an active thermal protection system, Int. J. Heat Mass Transfer 34 (3) (1991) 625-632.

[4] S. Maruyama, R. Viskanta, T. Aihara, Analysis of an active high-temperature thermal insulation system, Int. J. Heat Fluid Flow 11 (3) (1990) 196-203.

[5] S. Maruyama, Radiation heat-transfer control by a porous layer and a gas injection, Trans. JSME, Ser. B 58 (545) (1991) 211-215.

[6] S. Maruyama, N. Shimizu, Temperature distribution in a layer of an active thermal insulation system heated by a gas burner, Heat Transfer-Jpn. Res. 22 (3) (1993) 222-237.

[7] T. Yano, M. Ochi, S. Enya, Protection against fire and high temperature by using porous media and water, in: Proc. ASME/JSME Thermal Eng. Joint Conference, vol. 4, 1991, pp. 213-218.

[8] D.A. Torvi, J.D. Dale, Heat transfer in thin fibrous materials under high heat flux, Fire Technol. 35 (3) (1999) 210-231.

[9] D.A. Torvi, G.V. Hadjisophocleous, Research in protective clothing for firefighters: State of the art and future direction, Fire Technol. 35 (2) (1999) 111-130.

[10] M.A. Azhakesan, T.J. Shields, G.W.H. Silkock, On the nature, influence and magnitudes of flame heat transfer during surface flame spread, Fire Safety J. 35 (3) (2000) 189-222.

[11] D. Mangan, When engines entrap, Wildfire (July/August) (2002) 18-21.

[12] D.A. de Vries, Simultaneous transfer of heat and moisture in porous media, Trans. Am. Geophys. Union 39 (1958) 909-916.

[13] S. Whitaker, Heat and mass transfer in granular porous media, Adv. Drying 1 (1980) 24-61. 
[14] A.V. Luikov, Systems of differential equations of heat and mass transfer in capillary-porous media, Int. J. Heat Mass Transfer 18 (1975) 1-14.

[15] J. van Brakel, Mass transfer in convective drying, Adv. Drying 1 (1980) 217-266.

[16] Y.K. Sherwood, Application of the theoretical diffusion equations to the drying of solids, Trans. Am. Inst. Chem. Engrs. 27 (1931) 190-202.

[17] G.R. Hadley, Theoretical treatment of evaporation front drying, Int. J. Heat Mass Transfer 25 (10) (1982) 1511-1522.
[18] G.E. Baukal, B. Gebhart, A review of empirical flame impingement heat transfer correlations, Int. J. Heat Fluid Flow 17 (4) (1996) 386-396.

[19] L.L. Dong, C.S. Cheung, C.W. Leung, Heat transfer from an impinging butane/air slot flame jet, Int. J. Heat Mass Transfer 45 (5) (2002) 979-992.

[20] R. Filipczak, R.E. Lyon, Heat flux measurements in the OSU rate of heat release apparatus, Fire Safety J. 37 (6) (2002) 591-604. 